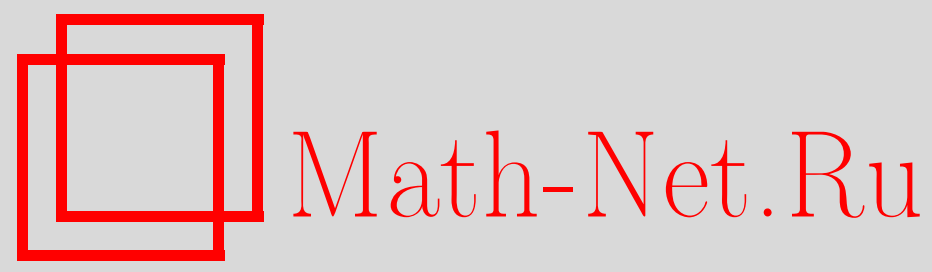

В. М. Храпченко, Упрощенное доказательство одной нижней оценки сложности, Дискрет. матем., 2013, том 25, выпуск $2,82-84$

DOI: https://doi.org/10.4213/dm1236

Использование Общероссийского математического портала Math-Net.Ru подразумевает, что вы прочитали и согласны с пользовательским соглашением http://www . mathnet.ru/rus/agreement

Параметры загрузки:

IP : 3.80 .181 .102

26 апреля 2023 г., $17: 29: 40$ 


\title{
Упрощенное доказательство одной нижней оценки сложности
}

\author{
(ㄷ) 2013 г. $\quad$ B. М. Храпченко
}

\begin{abstract}
Значительно упрощено доказательство одного известного неравенства, позволяющего получать квадратичные нижние оценки сложности П-схем (или, что то же самое, формул над базисом $\{\&, \vee,-\})$ для многих булевых функций.

Работа выполнена при финансовой поддержке Программы фундаментальных исследований ОМН РАН "Алгебраические и комбинаторные методы математической кибернетики проект "Синтез и сложность управляющих систем".
\end{abstract}

В работе [1] было доказано неравенство

$$
L\left(f_{n}\right) \geqslant \frac{|R|^{2}}{\left|M^{1}\right|\left|M^{0}\right|},
$$

где $f_{n}=f_{n}\left(x_{1}, \ldots, x_{n}\right)$ - произвольная булева функция (быть может, не всюду определенная), $L\left(f_{n}\right)$ - сложность минимальной (по числу контактов) П-схемы, реализующей $f_{n}, M^{1}$ - произвольное подмножество множества вершин $n$-мерного единичного куба (т. е. двоичных наборов), в которых функция $f_{n}$ равна $1, M^{0}-$ произвольное подмножество вершин $n$-мерного единичного куба, в которых функция $f_{n}$ равна $0, R$ - множество всех ребер куба, соединяющих вершины из $M^{1}$ с вершинами из $M^{0}$ (т. е. множество всех таких пар наборов $\left(\tilde{\sigma}^{1}, \tilde{\sigma}^{0}\right)$, что $\tilde{\sigma}^{1} \in M^{1}$, $\tilde{\sigma}^{0} \in M^{0}$ и наборы $\tilde{\sigma}^{1}$ и $\tilde{\sigma}^{0}$ отличаются друг от друга ровно в одном разряде, $|M|-$ мощность множества $M$.

Первоначальное доказательство (как это чаще всего и бывает) шло не по самому короткому пути и было перегружено деталями, не имеющими прямого отношения к основному ходу рассуждения.

Первый шаг по улучшению доказательства сделал В.В.Глаголев и сообщил свое наблюдение автору. Возможность дальнейшего упрощения заметил К.Л.Рычков и использовал это в работе [2]. Наконец, последний шаг сделал автор. Поскольку неравенство (1) включается в курсы лекций по дискретной математике и математической кибернетике, автор счел полезным опубликовать улучшенное доказательство.

В доказательстве неравенства (1) существенную роль играют два понятия, относящиеся к контактным схемам (в том числе и к П-схемам): общеизвестное понятие цепи (без самопересечений) и не так часто встречающееся понятие тупикового сечения. Стоит кое-что напомнить.

Множество контактов схемы называется сечением, если оно содержит хотя бы по одному контакту из каждой цепи этой схемы. Контактная схема проводит (реализуемая ею функция равна 1) на наборе $\tilde{\sigma}$ в том и только том случае, когда в ней есть 
цепь, все контакты которой замкнуты на этом наборе, и не проводит (реализуемая ею функция равна 0) на наборе $\tilde{\sigma}$ в том и только том случае, когда есть сечение схемы, все контакты которого разомкнуты на этом наборе. По аналогии с сечением цепь будет рассматриваться также и как соответствующее множество контактов. Наконец, сечение называется тупиковъм, если любое его собственное подмножество сечением не является.

В работе [3] доказана следующая

Лемма 1.. Во всякой П-схеме каждая иепь с каждым тупиковым сечением имеет ровно один общий контакт.

Лемма легко доказывается индукцией по числу контактов. При всей своей простоте она играет решающую роль в последующих рассуждениях.

Доказательство неравенства (1). Рассмотрим произвольную П-схему $S$, реализующую $f_{n}\left(x_{1}, \ldots, x_{n}\right)$. Число ее контактов (сложность) обозначим через $L$ и контакты занумеруем числами от 1 до $L$. Каждому набору $\tilde{\sigma}^{1} \in M^{1}$ сопоставим (какуюнибудь одну) цепь схемы $S$, все контакты которой замкнуты на этом наборе, а каждому набору $\tilde{\sigma}^{0} \in M^{0}$ сопоставим (какое-нибудь одно) тупиковое сечение схемы $S$, все контакты которого разомкнуты на этом наборе.

Построим таблицу размера $\left|M^{1}\right|\left|M^{0}\right| \times L$, каждая строка которой соответствует паре наборов $\left(\tilde{\sigma}^{1}, \tilde{\sigma}^{0}\right)$, где $\tilde{\sigma}^{1} \in M^{1}, \tilde{\sigma}^{0} \in M^{0}$, а $i$-й столбец соответствует $i$-му контакту. Заполнять таблицу будем постепенно. Для этого будем по очереди рассматривать ребра, соединяющие вершины из $M^{1}$ с вершинами из $M^{0}$, т. е. пары соседних (отличающихся в одном разряде) наборов $\left(\tilde{\sigma}^{1}, \tilde{\sigma}^{0}\right)\left(\tilde{\sigma}^{1} \in M^{1}, \tilde{\sigma}^{0} \in M^{0}\right)$. Каждая такая пара определяет цепь (сопоставленную набору $\tilde{\sigma}^{1}$ ) и тупиковое сечение (сопоставленное набору $\tilde{\sigma}^{0}$ ). Согласно лемме 1 , они имеют ровно один общий контакт. Пусть его номер будет $i$. Тогда на пересечении $i$-го столбца и строки, соответствующей паре $\left(\tilde{\sigma}^{1}, \tilde{\sigma}^{0}\right)$, ставится 1 .

После того, как будут просмотрены все ребра, соединяющие вершины из $M^{1}$ с вершинами из $M^{0}$, в таблице появится $|R|$ единиц. Обозначим число единиц в $i$-м столбце через $a_{i}$. Это число пар соседних наборов $\left(\tilde{\sigma}^{1}, \tilde{\sigma}^{0}\right)\left(\tilde{\sigma}^{1} \in M^{1}, \tilde{\sigma}^{0} \in M^{0}\right)$, для каждой из которых соответствующие цепь и тупиковое сечение пересекаются по $i$-му контакту. Покажем, что все наборы, входящие в эти пары, различны.

Пусть $i$-му контакту приписан символ $x_{j}^{\alpha}(j \in\{1, \ldots, n\}, \alpha \in\{0,1\})$. Тогда для любой из рассматриваемых пар $\left(\tilde{\sigma}^{1}, \tilde{\sigma}^{0}\right)\left(\tilde{\sigma}^{1} \in M^{1}, \tilde{\sigma}^{0} \in M^{0}\right)$ в соответствующей цепи на наборе $\tilde{\sigma}^{1}$ замкнуты все контакты и, стало быть, в $j$-м разряде набора $\tilde{\sigma}^{1}$ стоит $\alpha$, а в соответствующем тупиковом сечении на наборе $\tilde{\sigma}^{0}$ разомкнуты все контакты, и, стало быть, в $j$-м разряде набора $\tilde{\sigma}^{0}$ стоит $\bar{\alpha}$. Таким образом, по набору $\tilde{\sigma}^{1}$ из такой пары набор $\tilde{\sigma}^{0}$ определяется однозначно: в $j$-м разряде стоит противоположное значение, а во всех остальных разрядах значения совпадают. Аналогично в обратную сторону. Это означает, что наборы $\tilde{\sigma}^{1}$ и $\tilde{\sigma}^{0}$ в этом множестве пар встречаются по одному разу.

Итак, имеется $a_{i}$ наборов $\tilde{\sigma}^{1}$, для которых соответствующая цепь содержит $i$-й контакт, и $a_{i}$ наборов $\tilde{\sigma}^{0}$, для которых соответствующее тупиковое сечение содержит $i$-й контакт. Объединяя каждый из рассматриваемых наборов $\tilde{\sigma}^{1}$ с каждым из рассматриваемых наборов $\tilde{\sigma}^{0}$, получим $a_{i}^{2}$ пар, обладающих свойством: цепь и тупиковое сечение, соответствующие паре, пересекаются по $i$-му контакту. Против каждой такой пары в $i$-м столбце таблицы поставим 1 (если там единицы еще не было). В результате в $i$-м столбце таблицы будет $a_{i}^{2}$ единиц. 
Заметим, что

$$
\sum_{i=1}^{L} a_{i}=|R|
$$

а число единиц в таблице равно

$$
\sum_{i=1}^{L} a_{i}^{2}
$$

Поскольку цепь и тупиковое сечение имеют ровно один общий контакт, в каждой строке таблицы содержится не более одной единицы, а значит, общее число единиц в таблице не больше числа ее строк:

$$
\sum_{i=1}^{L} a_{i}^{2} \leqslant\left|M^{1}\right|\left|M^{0}\right|
$$

Пользуясь неравенством Коши-Буняковского, получаем:

$$
|R|^{2}=\left(\sum_{i=1}^{L} a_{i} \cdot 1\right)^{2} \leqslant\left(\sum_{i=1}^{L} a_{i}^{2}\right) \underbrace{\left(1^{2}+\ldots+1^{2}\right)}_{L} \leqslant\left|M^{1}\right|\left|M^{0}\right| L .
$$

Ввиду произвольности П-схемы $S$ отсюда следует неравенство (1).

\section{Список литературы}

1. Храпченко В. М., Об одном методе получения нижних оценок сложности П-схем. Матем. заметки (1971) 10, №1, 83-92.

2. Рычков К. Л., Модификация метода В. М. Храпченко и его применение к оценке сложности П-схем для кодовых функций. Методы дискретного анализа в теории графов и схем (1985) 42, 91-98.

3. Храпченко В. М., О сложности реализации линейной функции в классе П-схем. Матем. заметки (1971) 9, №1, 35-40.

Статья поступила 19.12.2012. 